\title{
PERCEPÇÃO DOS USUÁRIOS SOBRE A TRIAGEM COM CLASSIFICAÇÃO DE RISCO EM UM SERVIÇO DE URGÊNCIA DE CABO VERDE
}

\section{Users'perception of the risk assessment screening in an emergency care service in Cape Verde}

\section{Percepción de los usuarios sobre la selección de pacientes con clasificación de riesgo de un servicio de urgencia de Cabo Verde}

\author{
Regina Stella Spagnuolo \\ Universidade Estadual Paulista - UNESP - Botucatu (SP) - Brasil
}

Maria Natalina Lopes Silva

Hospital Agostinho Neto - Cidade da Praia - Cabo Verde

\section{Silmara Meneguin}

Universidade Estadual Paulista - UNESP - Botucatu (SP) - Brasil

Jamile Gabriela Bronzato Bassetto

Universidade Estadual Paulista - UNESP - Botucatu (SP) - Brasil

Violeta Campolina Fernandes

Universidade Estadual Paulista - UNESP - Botucatu (SP) - Brasil

\section{RESUMO}

Objetivo: Objetivou-se desvelar as concepções dos usuários acerca da triagem com classificação de risco em um serviço de urgência. Métodos: Trata-se de uma pesquisa qualitativa, alicerçada no método "estudo de caso", realizada com 17 usuários do Serviço de Urgência do Adulto (SUA) do Hospital Agostinho Neto, na ilha de Cabo Verde, África. Foram realizadas entrevistas abertas de agosto a outubro de 2012, e os dados analisados à luz da análise de conteúdo representacional temática. Resultados: Revelaram-se três categorias temáticas: 1) "Triagem prévia pelo enfermeiro"; 2) "Serviço de urgência: atendimento rápido para salvar vidas" 3) "Desconhecimento do propósito de classificação de risco". Conclusão: Concluiu-se que os usuários possuem pouco conhecimento sobre a triagem com classificação de risco, o que pode contribuir para a superlotação e prejudicar o atendimento dos casos considerados urgentes.

Descritores: Emergências; Serviços Hospitalares; Acolhimento.

\section{ABSTRACT}

Objective: To reveal users 'perception of the risk assessment screening in an emergency service. Methods: Qualitative case study of 17 users of the Adult Emergency Department (AED) of the Agostinho Neto Hospital located in Cape Verde, Africa. Open interviews were carried out from August to October 2012 and the data were analyzed using the thematic content analysis. Results: Three thematic categories emerged: 1) "Prior screening by nurses"; 2) "Emergency service: quick response to save lives"; 3) "Unawareness of the purpose of risk assessment". Conclusion: Users have little knowledge of the risk assessment screening, which may contribute to overcrowding and affect the care of cases considered urgent.

Descriptors: Emergencies; Hospital Services; User Embracement.

\section{RESUMEN}

Objetivo: Desvelar las concepciones de los usuarios sobre la selección de pacientes con clasificación de riesgo de un servicio público de urgencias. Métodos: Se trata de una investigación cualitativa con el método de "estudio de caso" realizada con 17 usuarios del Servicio de Urgencias del Adulto (SUA) del Hospital Agostinho Neto en la isla de Cabo Verde, África. Fueron realizadas entrevistas abiertas entre agosto y octubre de 2012 y los datos fueron analizados a la luz del análisis de contenido por temáticas. Resultados: Se revelaron tres categorías temáticas: 1) "Selección previa del enfermero"; 2) "Servicio de urgencias: atención rápida para salvar vidas" 3) "Desconocimiento del propósito de la clasificación de riesgo". Conclusión: Se concluye que los usuarios tienen poco conocimiento sobre la selección de pacientes con clasificación de riesgo lo que puede contribuir para la superlotación y perjudicar la atención de los casos que son considerados urgentes.

Descriptores: Urgencias Médicas; Servicios Hospitalarios; Acogimiento. 


\section{INTRODUÇÃO}

A triagem com classificação de risco é compreendida como um modo de operar os processos de trabalho em saúde, assumindo uma postura capaz de acolher, escutar e dar respostas adequadas aos pacientes, ou seja, requer prestar um atendimento com responsabilização e resolutividade, e, quando for o caso, orientá-los, bem como sua família, para a continuidade da assistência em outros serviços. Para tanto, requer o estabelecimento de referência e contrareferência com esses serviços para garantir a eficácia desses encaminhamentos ${ }^{(1)}$.

A classificação de risco configura-se como um método de seleção que tem por finalidade redirecionar o cliente conforme a prioridade de atendimento, de forma humanizada, informando o seu tempo de espera, organizando o fluxo de pacientes no sistema de saúde, selecionando os meios adequados para o diagnóstico e o tratamento do problema de saúde apresentado ${ }^{(2)}$.

Acolhimento com classificação de risco é um processo adaptado como sinônimo de triagem, e deve ser dinâmico, contínuo, devendo incluir atividades que acalmam os pacientes e seus familiares, oferecendo-lhes apoio emocional e segurança ${ }^{(3)}$.

$\mathrm{Na}$ busca de soluções para a superlotação desses serviços e na expectativa de assegurar o atendimento aos pacientes graves frente a reduzida eficiência e dificuldade para atender a população em suas necessidades, diversos países implantaram sistemas ou prototocolos de avaliação e estratificação de risco ${ }^{(4)}$.

Urgência é definida como "uma situação de agravo à saúde, com ou sem risco potencial de vida, cujo paciente depende de assistência médica"(5). Os serviços de urgência devem estar organizados para prestar uma adequada assistência a indivíduos em situações de urgência e emergência, que caracterizam, respectivamente, em situações que não há risco iminente de morte, mas necessitam atendimento rápido, e situações que requerem atendimento imediato e há risco de morte ${ }^{(6)}$.

Sabe-se que os serviços de urgência são pilares importantes da assistência à saúde em Cabo Verde e ainda utilizados como portas de entrada do sistema de saúde. Cabo Verde é um país jovem, localizado no Oceano Atlântico e constituído por 10 ilhas à noroeste da costa Africana. Possui uma população de aproximadamente 500 mil habitantes, dos quais 54,4\% possuem menos de 25 anos. Está entre os países que apresenta os melhores indicadores de saúde, devido a um esforço governamental e parcerias internacionais desde a sua independência recente, o que tem proporcionado melhoria da infraestrutura, formação de recursos humanos e estruturação dos serviços ${ }^{(7)}$.

Nos ultimos anos, os serviços de saúde do país sofreram consideráveis transformações, condicionadas pelas modificações nos modelos de gestão e organização das Redes de cuidados da saúde, o que possibilitou o aumento da qualidade dos cuidados prestados à população e também a melhoria do acesso ${ }^{(7,8)}$.

Todavia, os cidadãos cabo-verdianos permanecem "insatisfeitos com a prestação do Serviço Nacional de Saúde, com as situações de atendimento nas instituições públicas dos distintos níveis e com a qualidade e eficácia dos cuidados prestados" (9).

A triagem no serviço de urgência do adulto (SUA) do Hospital Agostinho Neto (HAN), único na Ilha de Santiago, cidade de Praia (capital), Cabo Verde, tem sido praticada há vários anos por médicos e enfermeiros, mas sem um critério clínico determinado que sustente um atendimento organizado, com qualidade, principalmente no que se refere à humanização e ao acolhimento. Face a esse problema, em 2012 foi proposta a implementação de um protocolo de triagem com classificação do risco por gravidade com cinco cores, sustentado pelo protocolo de Manchester ${ }^{(10)}$, sendo: vermelha para grau I, laranja para grau II, amarela para grau III, verde para grau IV e azul para grau V. Vale destacar que os três primeiros graus representam os mais urgentes e prioritários para atendimento, com tempo de espera decrescente a partir do grau $\mathrm{I}^{(10,11)}$.

Diante desse cenário, objetivou-se desvelar as concepções dos usuários acerca da triagem com classificação de risco em um serviço de urgência.

\section{MÉTODOS}

Trata-se de estudo de natureza qualitativa, de caráter descritivo, alicerçado no método "estudo de caso", realizado com usuários do Serviço de Urgência do Adulto (SUA) do Hospital Agostinho Neto da cidade de Praia, Ilha de Santiago, Cabo Verde, África.

O hostpital que foi cenário da pesquisa, caracterizado como geral, localizado no centro histórico da capital, é considerado como referência para a população que requer atendimento clínico e cirúrgico. É uma instituição pública, sob tutela do Ministério da Saúde, e possui 348 leitos e tem como retaguarda o hospital Trindade, onde são prestados os atendimentos e internações aos usuários psiquiátricos. Cabe esclarecer que, em Cabo Verde, o acesso aos serviços de saúde é tributado por meio de uma taxa denominada "coparticipação", que representa um pagamento pela assistência requerida.

Os participantes do estudo foram usuários do serviço de urgência do adulto, do município de Praia, Ilha Santiago, Cabo Verde - África, que concordaram em participar da pesquisa e assinaram o Termo de Consentimento Livre e Esclarecido (TCLE).

A coleta de dados ocorreu no período de julho a outubro de 2012, nos períodos matutinos, vespertinos e noturnos, visando abarcar diferentes momentos e percepções dos usuários. Foram adotados os seguintes critérios de inclusão: usuários que procuraram o SUA com idade superir a 18 anos e que estavam na classificação de risco ("amarela", "verde” ou "azul”). Os 
critérios de exclusão foram usuários que apresentavam as categorias de triagem ("vermelha" ou "laranja"). O presente estudo foi realizado durante o processo de implantação do Protocolo de Classificação de Risco no Hospital Dr. Agostinho Neto (HAN).

Como estratégia, utilizou-se entrevistas abertas norteadas pelas questões: Conte-me, o que o Sr.(a) entende por triagem? O que é atendimento de urgência para o Senhor(a)?

A entrevista aberta é utilizada quando o pesquisador deseja obter o maior número possível de informações sobre determinado tema segundo a visão do entrevistado, e também para obter um maior detalhamento do assunto em questão. Ela é utilizada geralmente na descrição de casos individuais, na compreensão de especificidades culturais para determinados grupos e para comparabilidade de diversos $\operatorname{casos}^{(12)}$. As entrevistas foram áudio gravadas, transcritas e posteriormente eliminadas.

A amostragem se deu por saturação teórica na $17^{\mathrm{a}}$ entrevista, momento em que as narrativas dos participantes tornaramse repetitivas. Entende-se por saturação teórica a suspensão de novas entrevistas quando, para o pesquisador, há repetição de material suficiente e redundante nos discursos, podendo ser interrompida a coleta, assim novos dados deixam de surgir ${ }^{(13)}$. Para este estudo não foi realizado projeto-piloto.

As entrevistas duraram aproximadamente 15 minutos cada uma, e cada entrevistado recebeu um código de identificação com a intenção de preservar sua privacidade e individualidade. Cada código consiste em uma combinação de dois caracteres, sendo cada caractere separado por um ponto: o primeiro caractere consiste na letra U significando que o entrevistado é um usuário do SUA e o segundo caractere consiste em uma variável numérica apresentando a sequência das entrevistas (U1, U2, etc). Para tratamento e análise dos dados, utilizou-se a análise de conteúdo categorial ${ }^{(14)}$.

O estudo foi aprovado pelo único Comitê de Ética do país (Ministério da Saúde de Cabo Verde), obtendo parecer favorável

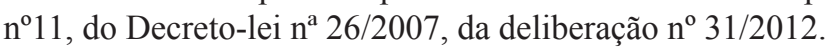

O Programa Internacional de Apoio à Pesquisa e ao Ensino por meio da mobilidade docente e discente internacional entre universidades de países de língua portuguesa, teve como meta a implantação de um curso de mestrado em Saúde Pública e linhas de pesquisa na Universidade Cabo-Verdiana, com a participação de docentes da universidade brasileira e profissionais da saúde local na condição de alunos, resultando na presente investigação como fruto desse processo.

\section{RESULTADOS}

Dos 17 participantes, sete eram do sexo masculino e 10 do sexo feminino, com idades compreendidas entre 14 e 64 anos.

A partir da análise dos depoimentos, emergiram três categorias temáticas: 1) "Triagem prévia pelo enfermeiro"; 2) "Serviço de urgência: atendimento rápido para salvar vidas" 3) "Desconhecimento do propósito de classificação de risco".

Em relação aos resultados da categoria 1, os usuários do SUA do Hospital Agostinho Neto, entendem que triagem é passar por atendimento de enfermagem antes de passar por atendimento médico. Na ocasião são verificados sinais vitais, coletam-se as queixas do momento, anota-se no prontuário e encaminha-se ao médico conforme os fragmentos dos discursos abaixo:

"Triagem é passar pelo enfermeiro antes do médico, dizer o que está a sentir para o enfermeiro, e ele escreve e manda para o médico” (U1).

"Ser atendido pelo enfermeiro antes de ir para o médico" (U4).

"Entendo que é uma intervenção feita antes de chegar ao médico e que pode ser feito por enfermeiro ou agente de saúde" (U6).

Para os participantes, a triagem tem sido entendida como uma pré-consulta, em que os enfermeiros fazem perguntas e anotações para posterior atendimento médico, como se resultasse em uma atividade desenvolvida para auxiliar o atendimento médico.

No que diz respeito à categoria 2, os participantes consideram que todo usuário que adentra ao espaço físico hospitalar da urgência tem risco de vida e necessita de atendimento imediato. O espaço físico de um serviço de urgência tem uma representação importante no imaginário dos usuários, isto é, o atendimento de urgência é parte integrante de uma estrutura física onde inicialmente são avaliados os parâmetros bioquímicos e sinais vitais para serem classificados, ou não, como casos urgentes e encaminhados para o atendimento proposto segundo a classificação de risco.

"[...] é quando alguém está a sentir que está a morrer, então tem que passar para urgência, para receber um atendimento rápido" (U1).

"É aqui onde eu estou agora, o lugar com atendimento mais rápido” (U3).

"Quando alguém apresenta, por exemplo, ferida, queda e que deve ter um atendimento mais rápido” (U4).

"Entendo que é o local de urgência onde é verificada a pressão e temperatura, se não for grave, e eles solicitam exames. Eu acho que qualquer pessoa que vai a urgência é porque precisa ser visto com urgência nesse local” (U7).

"Quando paciente apresenta um estado grave e que tem prioridade no atendimento" (U12).

"É rapidez, casos mais urgentes (...)" (U14). 
Os resultados da categoria 3 revelaram que a triagem com classificação de risco ainda é desconhecida para a população, isto é, os participantes da pesquisa alegam falta de informação quando as cores são atribuídas e distribuídas, por exemplo:
"Me foi atribuida a cor azul, desisti da consulta e fui embora, não sabia do que se tratava" (U7).
"Se o enfermeiro explicasse bem a triagem, não teria esperado das 8:00 às 13h30, sendo que depois fui encaminhado pelo médico para consulta no centro de saúde" (U17).

\section{DISCUSSÃO}

No âmbito da saúde, o acolhimento com classificação e avaliação de risco é compreendido como uma ação técnicoassistencial, que supõe antecipadamente uma mudança na relação entre usuário e o profissional, colocando o primeiro como participante ativo no processo de produção de saúde(15).

No contexto dos serviços de urgência e emergência, onde os mesmos se deparam com grandes filas de espera e sem critérios, exceto o da hora de chegada, a distinção de riscos ou graus de sofrimentos se faz necessário para que todos sejam acolhidos. A adoção de um sistema de classificação de risco no acolhimento assegura o atendimento de acordo com a condição de saúde, apresentada na avaliação inicial realizada pelo profissional enfermeiro na triagem. Além disso, o paciente que não corre risco imediato, assim como a seus familiares, são informados sobre o tempo provável de espera. Nessa atividade, a triagem apresenta-se como um trabalho essencial para o correto redirecionamento dos pacientes, ao mesmo tempo em que tem como finalidade descongestionar o serviço de urgência e emergência. No entanto, deve ser realizada por profissionais qualificados, aumentando a resolutividade dos problemas e a qualidade do atendimento ${ }^{(16)}$.

No local onde foi realizada a pesquisa é fato que essa atividade, recém-implantada, é realizada por enfermeiros capacitados que, juntamente com o médico, constituem a equipe do SUA. Vários estudos têm sido feitos com a finalidade de compreender se existem benefícios na realização de triagem por enfermeiros ou médicos. No entanto, a literatura tem mostrado que o enfermeiro é o profissional de saúde considerado de excelência para o desempenho dessa atividade ${ }^{(3)}$.

Os enfermeiros reúnem as condições necessárias para a triagem, por possuirem uma linguagem clínica orientada para os sinais e os sintomas, e não para os diagnósticos, conseguindo estabelecer uma relação empática fundamental na minimização de sentimentos como ansiedade, agressividade ou impaciência, e explicando calmamente ao utente o objetivo do processo de triagem. Para além disso, os enfermeiros têm uma visão da globalidade do serviço e dos recursos neles existentes ${ }^{(17)}$.

A distribuição e concentração de profissionais de saúde nos hospitais atraem a população para as estruturas com maior número de recursos humanos qualificados e tecnológicos, ficando os centros de saúde à margem da procura pela população. A procura dos serviços de urgência, muitas vezes, é motivada pela imagem construída em relação aos mesmos, impondo-lhes a capacidade de rapidez, acolhimento e resolubulidade. Dessa forma, por apresentar acesso 24 horas por dia e profissionais qualificados, desvelou-se na atual pesquisa que o SUA é a porta de entrada para avaliação de parâmetros vitais e exames complementares.

Essa percepção vai ao encontro das preocupações contempladas no Plano Nacional de Saúde de Cabo Verde, que tem como meta reorganizar a atenção básica do serviço nacional de saúde, melhorar e dar condições de acesso às populações ${ }^{(9)}$.

Essa realidade é corroborada pela dificuldade de encaminhamentos para outras unidades básicas e nos agendamentos de consulta para as especialidades, o que contribui para a insatisfação dos usuários e superlotação do SUA. Um estudo apontou que a insatisfação dos usuários em relação aos cuidados de saúde estão relacionados ao tempo de espera $(51,3 \%)$, insuficiência de pessoal qualificado $(10,2 \%)$ e zona de residência da população, com os habitantes do centro urbano apresentando-se mais insatisfeitos $(62,4 \%)$ do que os da zona rural $(38,6 \%)^{(18)}$.

Esse resultado se alinha a outros estudos, concluindo-se que o atendimento nestes serviços estão relacionadas a aspectos como satisfação, rapidez no atendimento, resolubilidade e humanização. Contudo, pesquisas ressaltaram insatisfação quanto aos encaminhamentos para outras unidades de saúde e demora nos agendamentos de consulta para especialidades ${ }^{(19)}$, isto é, a maioria dos usuários desses serviços de urgência os procuram para a realização de exames e encaminhamentos para especialidades em decorrência da demanda reprimida para agendamentos e consultas nas unidades de saúde da atenção primária ${ }^{(20)}$.

Em um estudo sobre a avaliação dos serviços hospitalares de emergência do estado do Rio de Janeiro, Brasil, observou-se que a principal causa de superlotação nos 24 serviços de urgência e emergência foi o atendimento aos pacientes com problemas ambulatoriais. Em $60 \%$ desses, houve referência espontânea à falência da rede básica como determinante da superlotação ${ }^{(21)}$.

Essa superlotação também conduz o processo de trabalho para uma sobrecarga e falta de tempo para tomada de decisões. São frequentes as pressões por atendimentos rápidos, ficando a divisão do trabalho prejudicada ${ }^{(22)}$.

A utilidade do serviço de triagem está alicerçada especialmente em prevenir complicações e reconhecer quadros agudos, que provocam risco de morte aos usuários. Para o melhor funcionamento desse serviço é necessária a sua integração aos outros serviços de saúde existentes no sistema, estabelecendo vínculos com os mesmos e permitindo o adequado encaminhamento dos usuários $^{(19)}$. 
A implementação de triagem com avaliação e classificação de risco em Cabo Verde é uma estratégia de gestão nova no país, que demanda reorganização no âmbito nacional e municipal, além de novos estudos que contemplem todas as interfaces desse processo. O resultado da presente pesquisa foi encaminhado ao Ministério de Saúde de Cabo Verde para fortalecer o acolhimento com classificação de risco no SUA do Hospital Agostinho Neto e outros serviços semelhantes em Cabo Verde, visando a reorganização da assistência. Também foi validado pelos enfermeiros triadores em oficinas de trabalho visando o conhecimento das concepções dos usuários acerca da triagem e da urgência.

\section{CONCLUSÃO}

Concluiu-se que os usuários possuem pouco conhecimento sobre a triagem com classificação de risco, o que pode contribuir para a superlotação e prejudicar o atendimento dos casos considerados urgentes.

Devido à recente implantação dos serviços de triagem com classificação de risco em Cabo Verde, sugere-se a reorganização do sistema de referência e contrarreferência, além da criação de protocolos no sentido de diminuir a insatisfação dos usuários acerca dos serviços.

\section{AGRADECIMENTOS}

Os autores agradecem à Capes pelo apoio ao projeto em que este trabalho se insere.

\section{REFERÊNCIAS}

1. Ministério da Saúde (BR), Secretaria de Atenção à Saúde Política Nacional de Humanização da Atenção e Gestão do SUS. Acolhimento e Classificação de Risco nos Serviços de Urgência. Brasília: Ministério da Saúde; 2009.

2. Moura AC, Lopes AMS, Portela ALF, Sousa ID, Sousa SNS. Implantação do acolhimento com classificação de risco: um relato de experiência. Rev Multop Saúde HSM. 2013;1(2):72-82.

3. Zem KKS, Montezeli JH, Peres AM. Acolhimento com classificação de risco: concepção de enfermeiros de um pronto socorro. Rev RENE. 2012;13(4):899-08.

4. Oliveira GN, Vancini-Campanharo CR, Batista REA. Acolhimento com avaliação e classificação de risco: concordância entre os enfermeiros e o protocolo institucional. Rev Latinoam Enferm. 2013;21(2):500-6.

5. Martins, H, Zamboni V, Velasco IT. Atualização em emergências. São Paulo: Manole; 2009.

6. Oharal R, Melo MRAC, Lausl AM. Caracterização do perfil assistencial dos pacientes adultos de um pronto socorro. Rev Bras Enferm. 2010;63(5):749-54.

7. Ministério de Saúde (CV). Plano Nacional de desenvolvimento sanitário 2012-2016: reformar para uma melhor saúde. Praia; 2012. v. 1.

8. Ministério de Saúde (CV). Relatório Estatístico 2011. Praia; 2012.

9. Ministério de Saúde (CV). Politica Nacional de Saúde: reformar para uma melhor saúde. Praia; 2007 [acesso em 2016 Jun 17]. Disponível em: \%(http://www.governo.cv/documents/POLITINACIONALSAUDE_2020\%20\%282\%29.pdf

10. Coutinho AAP, Cecílio LCO, Mota JAC. Classificação de risco em serviços de emergência: uma discussão da literatura sobre o Sistema de Triagem de Manchester. Rev Méd Minas Gerais. 2012;22(2):188-98.

11. Freitas P. Triagem no serviço de urgência: grupo de triagem de Manchester. $2^{\mathrm{a}}$ ed. Portugal: BMJ Publishing Group; 2002.

12. Minayo M. O desafio do conhecimento: pesquisa qualitativa em Saúde. 13 ed. Hucitec: São Paulo; 2014.

13. Fontanella BJ, Ricas J, Turato E. Saturation sampling in qualitative health research: theoretical contributions. Cad Saúde Pública. 2008;24(1):17-27.

14. Bardin L. Análise de conteúdo. $7^{\text {a }}$ ed. Lisboa: Edições 70; 2011.

15. Bellucci JA Júnior, Matsuda LM. Implantação do sistema acolhimento com classificação e avaliação de risco e uso do fluxograma analisador. Texto \& Contexto Enferm. 2012;21(1):217-25.

16. Souza CC, Toledo AD, Tadeu LFR, Chianca TCM. Risk classification in a emergency room: agreement level between a brazilian institucional and the Manchester Protocol. Rev Latinoam Enferm. 2011;19(1):26-33. 
17. Caveião C, Hey AP, Montezeli JH, Barros APMM, Sordi JA, Santos SC. Desafios ao enfermeiro na implantação da classificação de risco em unidade mista. Rev Enferm. 2014;4(1):189-96.

18. Valdez TAS. Regionalização e integração sistêmica: cenários para a reforma do Sistema de Saúde de Cabo Verde [dissertação]. Rio de Janeiro: FIOCRUZ; 2007.

19. Azevedo J, Barbosa M. Triagem em serviços de saúde: percepções dos usuários. Rev Enferm. 2012;15(1):33-9.

20. Rossaneis MA, Haddad MCL, Borsato FG, Vannuchi MO, Sentoneet ADD. Caracterização do atendimento após implantação do acolhimento, avaliação e classificação de risco em hospital público. Rev Eletrônica Enferm. 2011;13(4):648-56.

21. O’Dower G, Matta IEA, Pepe VLE. Avaliação dos serviços hospitalares de emergência do Estado do Rio de Janeiro. Ciênc Saúde Coletiva. 2008;13(5):1637-48.

22. Lancman S, Gonçalves RMA, Mângia EF. Organização do trabalho, conflitos e agressões em uma emergência hospitalar na cidade de São Paulo, Brasil. Rev Ter Ocup Univ São Paulo. 2012;23(3):199-07.

\section{Endereço do primeiro autor:}

Regina Stella Spagnuolo

Faculdade de Medicina Campus de Botucatu

Av. Prof. Mário Rubens Guimarães Montenegro, s/n.

Bairro: UNESP - Campus de Botucatu

CEP. 18618687 - Botucatu - SP - Brasil

E-mail: rstella@fmb.unesp.br

\section{Endereço para correspondência:}

Maria Natalina Lopes Silva

Hospital Agostinho Neto

Largo Martires Pdjiguiti

Bairro: Plateau

CV - 7600 - Cidade da Praia - Cabo Verde

E-mail: natalinasilva1@hotmail.com 